\title{
Thermodynamic Origin of the Increased Rate of Hydrolysis of Phosphate and Phosphorothioate Esters in DMSO/Water Mixtures
}

\author{
Kerensa Sorensen-Stowell and Alvan C. Hengge \\ Contribution from the Department of Chemistry and Biochemistry, Utah State University, Logan, \\ Utah 84322-0300
}

\begin{abstract}
The hydrolysis rates of the dianions of phosphate and phosphorothioate esters are substantially accelerated by the addition of polar aprotic solvents such as DMSO and acetonitrile. The activation barrier $\Delta \mathrm{G}^{\ddagger}$ is smaller due to a lower enthalpy of activation. The enthalpy of transfer of $p$-nitrophenyl phosphate $(p \mathrm{NPP})$ and $p$-nitrophenyl phosphorothioate ( $p$ NPPT), from water to $0.6(\mathrm{~mol})$ aq DMSO (60 mol-percent water in DMSO) were measured calorimetrically. The enthalpies of activation for the hydrolysis reactions in the two solvents permitted the calculation of the enthalpy of transfer of the transition states. This transfer is thermodynamically favorable for both the reactants and the transition states, but is more favorable for the transition states. In the case of $p$ NPP, the enthalpy of transfer of the reactant is $-23.9 \mathrm{kcal} / \mathrm{mol}$, compared to -28.3 for the transition state. The difference is greater for $p$ NPPT, where the enthalpy of transfer of the reactant is $-23.2 \mathrm{kcal} / \mathrm{mol}$, and that for the transition state is -35.3 . The results show that the reduced enthalpies of activation in both hydrolysis reactions arise not from a destabilization of the reactants in the mixed solvent, but from the fact that the enthalpy of transfer of the transition states to the mixed solvent is significantly more negative than the enthalpy of transfer of the reactants.
\end{abstract}

\section{Introduction}

Phosphoryl transfer reactions are one of the most common reactions in biochemistry. The enzymatic hydrolysis of phosphate monoesters, most commonly tyrosine, serine, and threonine phosphates, is involved in cell signaling and regulation. The substrates for most phosphatases are believed to be the dianion forms, which have very slow rates of uncatalyzed hydrolysis; the estimated half-life for attack by water on alkyl phosphate dianions is $10^{12}$ years at $25^{\circ} \mathrm{C}$. $^{1}$

The rate of hydrolysis of phosphomonoester dianions is strongly affected by the medium. The rate of hydrolysis of the $p$-nitrophenyl phosphate ( $p$ NPP) dianion is accelerated by up to $10^{6}$ in a solution that is $95 \%$ DMSO, $5 \%$ water (which will be designated as 0.95 (v) aq DMSO), and similar rate accelerations occur in other mixed solvents. ${ }^{2}$ The hydrolysis of the phosphorothioate analog $p$-nitrophenyl phosphorothioate ( $p$ NPPT) is also accelerated by addition of DMSO, by nearly 7 orders of magnitude in $0.95(v)$ aq DMSO ${ }^{3}$ In both cases, the reduction in $\Delta \mathrm{G}^{\ddagger}$ arises from a sharply reduced enthalpy of activation in the mixed solvent. In the hydrolysis of the $p$ NPP dianion, $\Delta \mathrm{H}^{+}=30.6 \mathrm{kcal} / \mathrm{mol}$ in water, but is reduced to $20.7 \pm 0.6$ $\mathrm{kcal} / \mathrm{mol}$ in $0.95(v)$ aq DMSO. ${ }^{4}$ In the hydrolysis of $p$ NPPT, $\Delta \mathrm{H}^{\ddagger}$ is reduced from $37.0 \pm 1$ $\mathrm{kcal} / \mathrm{mol}$ in water to $22.9 \pm 0.7 \mathrm{kcal} / \mathrm{mol}$ in $0.95(v)$ aq DMSO. ${ }^{3}$ Similar considerations apply for the $p$ NPPT reaction, although the increases in pNPPT hydrolysis rates are not as dramatic at the highest DMSO percentages as for pNPP. ${ }^{3}$ 
In the original report ${ }^{2}$ of the medium effect on $p$ NPP hydrolysis, it was speculated that the rate acceleration might be due to the disruption of hydrogen bonding to the phosphoryl group, resulting in a weakening of the $\mathrm{P}-\mathrm{O}$ ester bond. This can be rationalized by a contribution from an extreme resonance form consisting of the phenolate ion and metaphosphate. The loss of stabilizing hydrogen bonds between the phosphoryl group and the solvent might be expected to enhance contribution from such a resonance form. The reduced enthalpy of activation is consistent, though not uniquely so, with a weaker scissile bond in the mixed medium. Similar kinetic isotope effects and Brønsted $\beta_{\lg }$ values ${ }^{4}$ indicate that the hydrolysis of $p$ NPP proceeds through a similar transition state in both solvent systems; thus, the rate acceleration does not result from a mechanistic change.

Subsequent experimental results have not supported the notion that DMSO or other solvents significantly weaken the scissile P-O ester bond. ${ }^{5,6}$ Cheng et al. ${ }^{5}$ used Raman spectroscopy on $p$ NPP in water/DMSO mixtures and determined that the bridging P-OR bond lengthens by only $\sim 0.013 \AA$ in 0.95 (v) aq DMSO. Further investigations using isotope shifts on ${ }^{31} \mathrm{P}$ NMR in various solvents ${ }^{6}$ found no evidence of significant weakening of the ester bond upon transfer from water to aprotic solvents. These results suggest that the rate increase arises from some other factor. One hypothesis is that the DMSO/water solvent mixture might better solvate the transition state, with its more dispersed charge, relative to the reactant. ${ }^{5}$ In order to test this hypothesis, the enthalpies of transfer for the transition states for the hydrolysis of $p$ NPP and of $p$ NPPT from water to a DMSO/water mixture were obtained, and compared with those of the reactants. Because the dominant contribution to the reduced free energy of activation in the mixed solvent is enthalpic, the enthalpies of transfer should be directly relevant to the source of the faster rate of reaction in water/DMSO mixtures.

\section{Results and Discussion}

The enthalpy of transfer of the transition state, $\delta \Delta \mathrm{H}^{\text {trans }}$, can be calculated from the enthalpy of transfer of the reactant and the enthalpy of activation:

$$
\delta \Delta \mathrm{H}^{\ddagger t r a n s}=\delta \Delta \mathrm{H}_{\mathrm{sol}}+\delta \Delta \mathrm{H}^{*}
$$

In this equation, $\delta \Delta \mathrm{H}_{\mathrm{sol}}$ is the difference between the enthalpy of solution of the reactant in the two solvents, which is the enthalpy of transfer. $\delta \Delta \mathrm{H}^{\ddagger}$ is the difference between the enthalpy of activation in the two solvents. A number of workers, particularly Haberfield, have obtained the enthalpy of solvent transfer of transition states for a number of reactions, and the notation in equation 1 follows these precedents ${ }^{7-12}$ The relationship in equation 1 is shown pictorially in Figure 1.

When the reactants are ionic, such as the phosphate ester dianions examined in this study, it is necessary to obtain the enthalpy of transfer of the reactant ion free of its counterions. This is accomplished using an extrathermodynamic assumption involving ion pairs consisting of two symmetric species, in which the central charge is shielded from solvent by a sizeable organic fragment. ${ }^{13}$ The enthalpy of solution $\left(\Delta \mathrm{H}_{\mathrm{sol}}\right)$ of such salts is assumed to consist of equal contributions from each ion. Tetrabutylammonium tetrabutylborate is one such commonly used pair, others being tetraphenylarsonium tetraphenylborate, and similar species. To obtain the enthalpy of solution for an anion using this method, the $\Delta \mathrm{H}_{\mathrm{sol}}$ of tetrabutylammonium tetrabutylborate is measured in the solvent of interest. Half of this quantity is assigned to the tetrabutylammonium ion. The $\Delta \mathrm{H}_{\mathrm{sol}}$ of the tetrabutylammonium salt of the anion of interest is then measured, and the contribution from the cation(s) is subtracted. Since its introduction by Grunwald, ${ }^{13}$ values of $\Delta \mathrm{H}_{\text {sol }}$ for a number of single ions have been obtained using this method. $7-12,14$ The enthalpy of transfer from one solvent to another is the difference between the enthalpies of solution in the two solvents of interest. 
Figure 1 shows one example of how a transfer from one solvent to another (from solvent 1 to solvent 2 in the example shown) would result in a reduced enthalpy of activation, if the enthalpy of transfer of the reactants is more thermodynamically unfavorable than the transfer of the transition state. The goal of this work was to determine if this, or some other scenario, occurs in the phosphate ester hydrolysis in the two solvents.

The first goal was to obtain enthalpies of solution of $p$ NPP and $p$ NPPT in water, and in aqueous DMSO. The solubility requirements to permit accurate calorimetric measurement of the enthalpy of solution of the bis(tetrabutylammonium) salts limited the DMSO composition of the mixture to $60 \% \mathrm{DMSO} / 40 \% \mathrm{H}_{2} \mathrm{O}$ by mole fraction $(0.6(\mathrm{~mol})$ aq DMSO). This corresponds to $0.85(v)$ aq DMSO. The mole fraction notation will be used to describe results in this solvent mixture, in keeping with the notation used in previous work in the same medium, some of which is referenced below.

The enthalpy of solution in $0.6(\mathrm{~mol})$ aq DMSO, and in water, for bis(tetrabutylammonium) $p$ NPP was measured and reported in Table 1. The bis(tetrabutylammonium) salt of $p$ NPPT could not be obtained. Phosphorothioates are isolated after synthesis as their cyclohexylammonium salts, but this salt form lacked sufficient solubility in the mixed medium to carry out calorimetry experiments. Attempts to convert $p$ NPPT to the bis (tetrabutylammonium) salt were accompanied by extensive hydrolysis, and pure samples could not be obtained (see Supplemental Information). The less reactive phenyl phosphorothioate (PPT) was successfully converted to the bis(tetrabutylammonium) salt, and calorimetry in water and the mixed solvent was carried out using this compound. Its oxygen analogue phenyl phosphate (PP) was also prepared and calorimetry carried out. The enthalpies of solution of these esters, and of the tetrabutylammonium salt of inorganic phosphate, are reported in Table 1. The magnitudes are in the range expected for polyvalent electrolytes. 15

To obtain the enthalpies of transfer $\left(\delta \Delta \mathrm{H}_{\mathrm{sol}}\right)$ of the anionic species from water to $0.6(\mathrm{~mol})$ aq DMSO, the contribution of the tetrabutylammonium ions were subtracted using the literature value for its enthalpy of transfer, ${ }^{7}$ to give the values in Table 2 . The exothermicity of the transfer of the phosphate esters and of inorganic phosphate, from water to the mixed solvent, was unexpected. It has long been known that the enthalpies of transfer from water to neat DMSO are exothermic for large, polarizable or delocalized anions such as iodide and perchlorate. ${ }^{14,15}$ The only anion for which enthalpy of transfer has been obtained for both neat DMSO and the mixed solvent used in the present study is iodide, for which the enthalpy of transfer to the mixed solvent $(-5.5 \mathrm{kcal} / \mathrm{mol})^{7}$ is more exothermic than for transfer to neat DMSO (reported values range from -2.6 to -4.9$).{ }^{15}$ The observation that inorganic phosphate follows the same trend demonstrates that the aromatic moiety is not responsible for the observed exothermicities in Table 2.

It is noted by comparing the data for phenyl and $p$-nitrophenyl phosphates, that there is a negligible difference in the heat of transfer imparted by the nitro group. Thus, it seems a safe assumption that the enthalpy of transfer for $p$ NPPT should closely approximate the value for PPT.

Because the activation parameters for the hydrolysis of $p$ NPP and $p$ NPPT have been reported in a 95\% (v) aq DMSO, somewhat different from the $0.6(\mathrm{~mol})$ aq DMSO used here, the rates of hydrolysis of these esters were measured as a function of temperature to construct Eyring plots (Figure 2), which were used to determine the activation parameters. The lines represent the best least-squares linear fit to the data. The slopes and intercepts were used to calculate the enthalpy and entropy of activation for each ester and their standard errors. These values along with literature values for the activation parameters for the hydrolysis reactions in water are reported in Table 3 . The activation parameters revealed a lower enthalpy of activation in 0.6 
$(\mathrm{mol})$ aq DMSO, as previously found for $0.95(\mathrm{v})$ aq DMSO. However, in the case of $p$ NPP, $\Delta \mathrm{H}^{\ddagger}$ is less dramatically reduced in $0.6(\mathrm{~mol})$ aq DMSO than when the reaction medium is 0.95 (v) aq DMSO. This observation is consistent with the previous finding that the hydrolysis rate of $p$ NPP increases most dramatically at very high DMSO percentage. ${ }^{2}$ The rate enhancement is $\sim 24$-fold upon increasing DMSO from $80 \%$ to $90 \%$ by volume, compared to an $\sim 1800$-fold enhancement caused by the change from $80 \%$ to $95 \%$ DMSO. ${ }^{16}$

The transition states for the hydrolysis reactions of both $p$ NPP and $p$ NPPT are loose, with little nucleophilic participation by water in the $p$ NPP reaction, and none in the case of $p$ NPPT, which reacts via a thiometaphosphate intermediate. ${ }^{18,19}$ The large, positive entropy of activation in the DMSO/water solvent indicates that the dissociative mechanism for $p$ NPPT hydrolysis is maintained in this solvent. Thus, the transition state resembles metaphosphate and the $p$ nitrophenoxide leaving group. KIEs and LFER data for the hydrolysis of both $p$ NPP and $p$ NPPT indicate that bond scission is far advanced, and that the phosphoryl group and the leaving groups share about a negative charge in the transition state. These transition states, with the respective charge distribution, are shown in Figure 3.

From the enthalpies of transfer and the corresponding enthalpies of activation, the enthalpies of transfer of the transition states of these two reactions can be calculated using Equation 1. The transfer of the $p$ NPP hydrolysis transition state from water to $0.6(\mathrm{~mol})$ aq DMSO is exothermic, $-28.3 \pm 0.5 \mathrm{kcal} / \mathrm{mol}$. Assuming the enthalpy of solution for $p$ NPPT is similar to that of PPT, a similar value for the $p$ NPPT transition state of $-31.2 \pm 1.2 \mathrm{kcal} / \mathrm{mol}$ is obtained. Figure 4 shows these relationships in pictorial fashion.

The above treatment omits consideration of the water nucleophile in the transition states of the rate-limiting steps of these reactions. While this is accurate in the dissociative mechanism for $p$ NPPT hydrolysis, a small amount of nucleophilic participation occurs in the hydrolysis of phosphomonoesters like $p$ NPP. The literature value for the enthalpy of transfer of water to 0.6 $(\mathrm{mol})$ aq DMSO is $-1.15 \mathrm{kcal} / \mathrm{mol}^{7}$ Inclusion of this quantity is shown in red in Figure 4 . The enthalpy of transfer for water plus $p \mathrm{NPP}^{2-}$ is more negative by $1.15 \mathrm{kcal} / \mathrm{mole}$, and as a result, the enthalpy of transfer of the transition state is more negative by the same amount.

These results show that the transfer of the transition state for the $p$ NPP and the $p$ NPPT hydrolysis reaction is more exothermic than for transfer of the respective reactant. Thus, this is the origin of the reduced enthalpy of activation in both reactions, rather than a scenario such as that outlined in Figure 1. In the case of $p$ NPP, the enthalpy of transfer of the reactant is -23.9 $\mathrm{kcal} / \mathrm{mol}$, compared to -28.3 for the transition state. The difference is greater for $p$ NPPT, where the enthalpy of transfer of the reactant is $-23.2 \mathrm{kcal} / \mathrm{mol}$, and -31.2 for the transition state. In each of these reactions the di-negative charge of the reactant becomes significantly more dispersed in the transition state, which probably accounts for the greater exothermicity of the transfer to $0.6(\mathrm{~mol})$ aq DMSO.

\section{Conclusions}

The results presented here, in conjunction with spectroscopic investigations on the effects of the solvent medium on P-O bonds in phosphate esters, indicate that the reduction in the enthalpy of activation for hydrolysis imparted by $0.6(\mathrm{~mol})$ aq DMSO arises from a more favorable enthalpy of transfer of the transition state relative to the enthalpy of transfer of the $p$ NPP or $p$ NPPT reactant. Due to experimental constraints that limited the percentage of DMSO in the solvent systems that could be examined, this work cannot exclude the possibility that additional effects might operate at higher DMSO fractions, where rate accelerations are more dramatic. 
These results demonstrate the potential for medium effects to significantly reduce the enthalpy of activation for phosphoryl transfer reactions. This provides a potential means for substantial rate accelerations to arise from electrostatic interactions in an enzymatic active site that stabilize the transition state for phosphoryl transfer. Due to the loose transition state for phosphomonoester reactions, the entropic barrier is small, leaving enthalpic considerations as the major avenue to achieve significant rate accelerations. The results here show that substantial reductions in the enthalpy of activation result upon the transfer from a medium of pure water to a $\mathrm{DMSO} /$ water mixture that remains $22 \mathrm{M}$ in water. This is probably modest in comparison to the environmental effects that can be achieved in the evolved active site of an enzyme.

\section{Experimental Section}

The synthesis and purification of $p$-nitrophenyl phosphate, phenyl phosphate, phenyl phosphorothioate, and $p$-nitrophenyl phosphorothioate were accomplished using literature methods, described in Supporting Information.

\section{Thermodynamic methods}

A commercial solution calorimeter was used to obtain the heats of solution of the phosphate and phosphorothioate esters in water, and in an aqueous DMSO mixture. The instrument was first standardized using tris(hydroxymethyl) aminomethane (TRIS), furnished with the calorimeter as a standardizing reagent, as described in Supporting Information. In a typical experiment, the heat evolved from solution of $250 \mathrm{mg}$ of solute in $100 \mathrm{~g}$ of solvent was measured, and used to obtain the enthalpy of solution. The difference between the enthalpy of solution in the two solvents is the enthalpy of transfer.

\section{Supplementary Material}

Refer to Web version on PubMed Central for supplementary material.

\section{Acknowledgements}

The authors thank the NIH for financial support (GM47297), and Tony Kirby for helpful discussions.

\section{References}

1. Lad C, Williams NH, Wolfenden R. Proc Natl Acad Sci U S A 2003;100:5607-10. [PubMed: 12721374]

2. Abell KWY, Kirby AJ. Tet Lett 1986;27:1085-1088.

3. Catrina IE, Hengge AC. J Am Chem Soc 1999;121:2156-2163.

4. Grzyska PK, Czyryca PG, Golightly J, Small K, Larsen P, Hoff RH, Hengge AC. J Org Chem 2002;67:1214-20. [PubMed: 11846665]

5. Cheng H, Nikolic-Hughes I, Wang JH, Deng H, O'Brien PJ, Wu L, Zhang ZY, Herschlag D, Callender R. J Am Chem Soc 2002;124:11295-306. [PubMed: 12236744]

6. Sorensen-Stowell K, Hengge AC. J Org Chem 2005;70:8303-8. [PubMed: 16209571]

7. Haberfield P. J Phys Chem 1983;87:1423-1426.

8. Haberfield P, Fortier D. J Org Chem 1983;48:4554-4557.

9. Haberfield P, Friedman J, Pinkston MF. J Am Chem Soc 1972;94:71-75.

10. Haberfield P, Kivuls J, Haddad M, Rizzo T. J Phys Chem 1984;88:1913-1916.

11. Haberfield P, Nudelman A, Bloom A, Romm R, Ginsberg H. J Org Chem 1971;36:1792-1795.

12. Haberfield P, Pessin J. J Am Chem Soc 1982;104:6191-6194.

13. Grunwald E, Baughman G, Kohnstam G. J Am Chem Soc 1960;82:5801-5811.

14. Fuchs R, Bear JL, Rodewald RF. J Am Chem Soc 1969;91:5797-5800. 
15. Criss, CM. Physical Organic Chemistry of Organic Solvent Systems. Covington, AK.; Dickinson, T., editors. Plenum; New York: 1973. p. 23-136.

16. Abell, KWY. PhD thesis. Cambridge University; 1984.

17. Kirby AJ, Varvoglis AG. J Am Chem Soc 1967;89:415-423.

18. Hengge, AC. Comprehensive Biological Catalysis: A Mechanistic Reference. Sinnott, M., editor. 1. Academic Press; San Diego, CA: 1998. p. 517-542.

19. Thatcher GRJ, Kluger R. Adv Phys Org Chem 1989;25:99-265. 


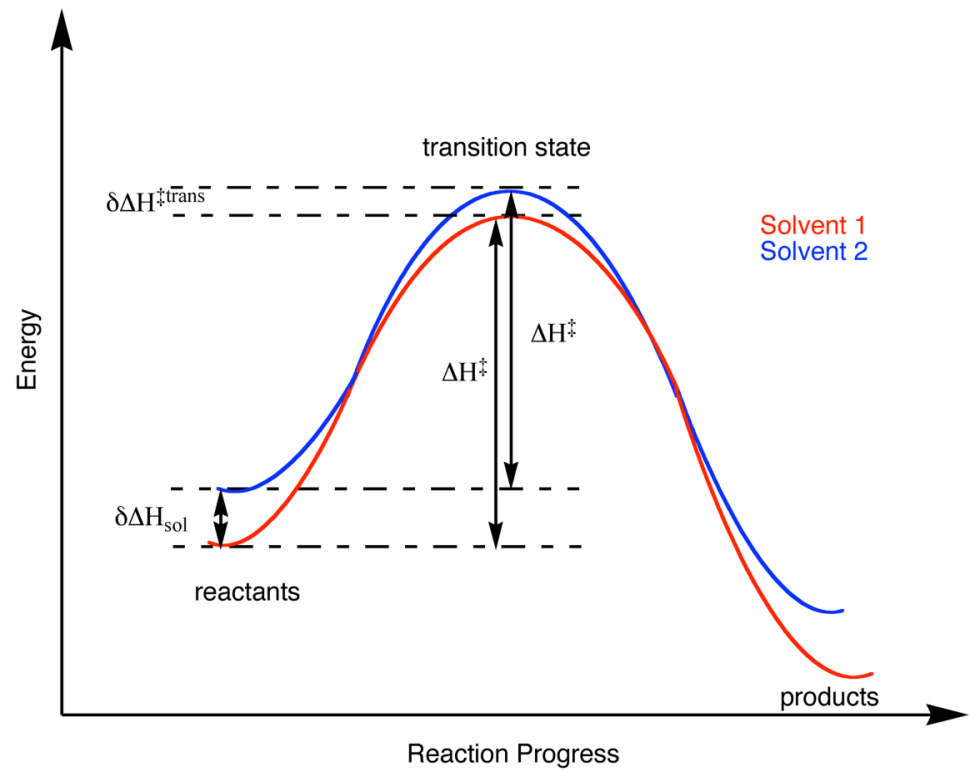

Figure 1.

Diagram of reaction coordinate showing the relationship between the thermodynamic parameters in Equation 1. 

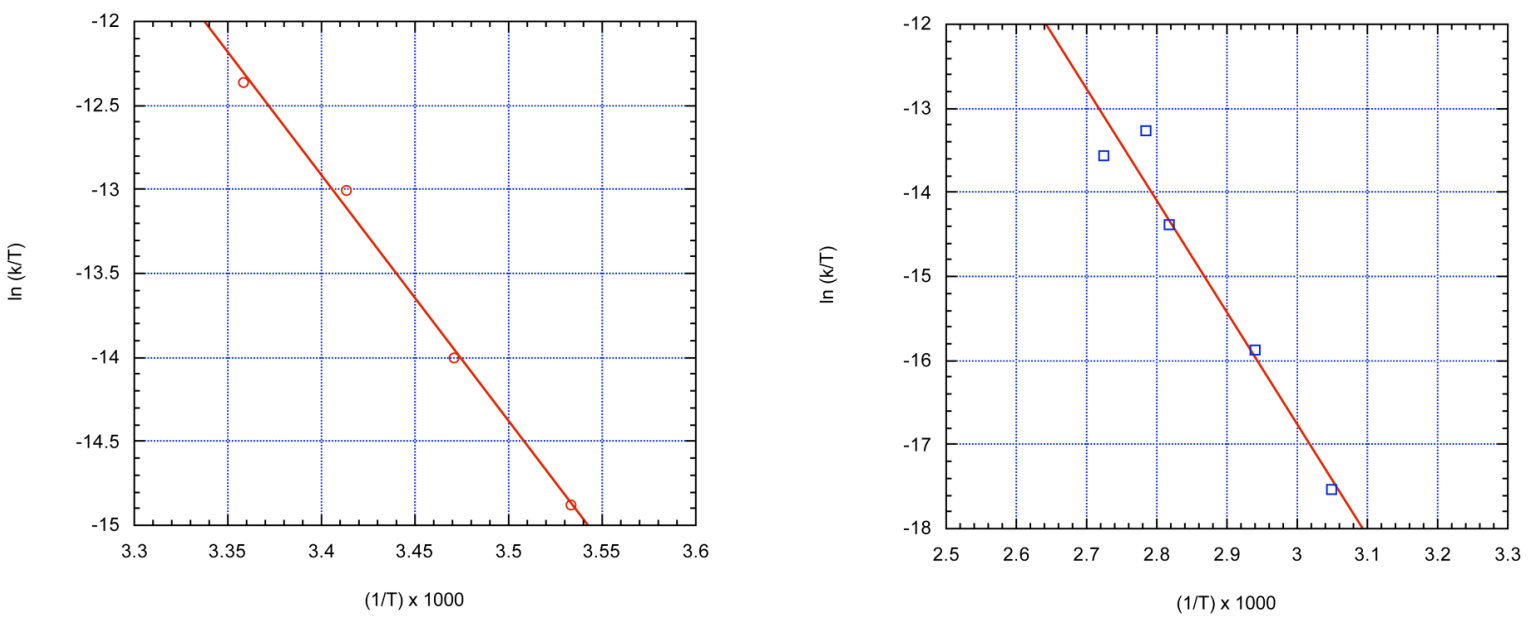

Figure 2.

Eyring plots for the hydrolysis reactions of $p$-NPPT $(\circ)$ and $p$-NPP $(\square)$ in $0.6(m o l)$ aq DMSO. 


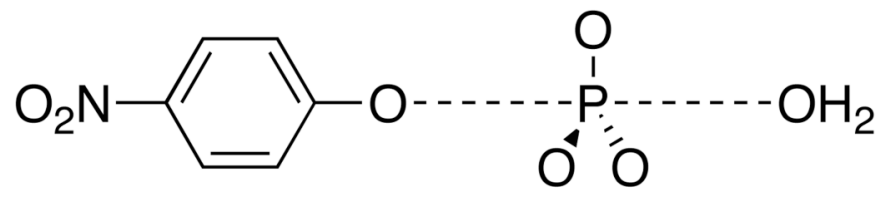

1- 1 -

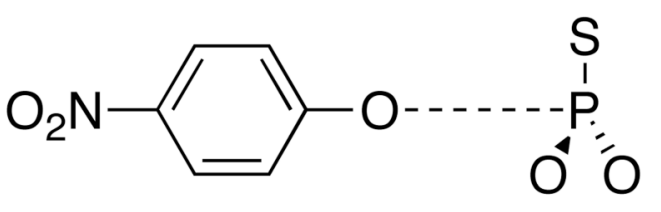

$1-$

$1-$

Figure 3.

Top, the transition state for $p$ NPP hydrolysis. The charge of -2 of the reactant is dispersed nearly equally between the leaving group and the phosphoryl group. The transition state for $p$ NPPT hydrolysis is similar, but lacks nucleophilic participation. 

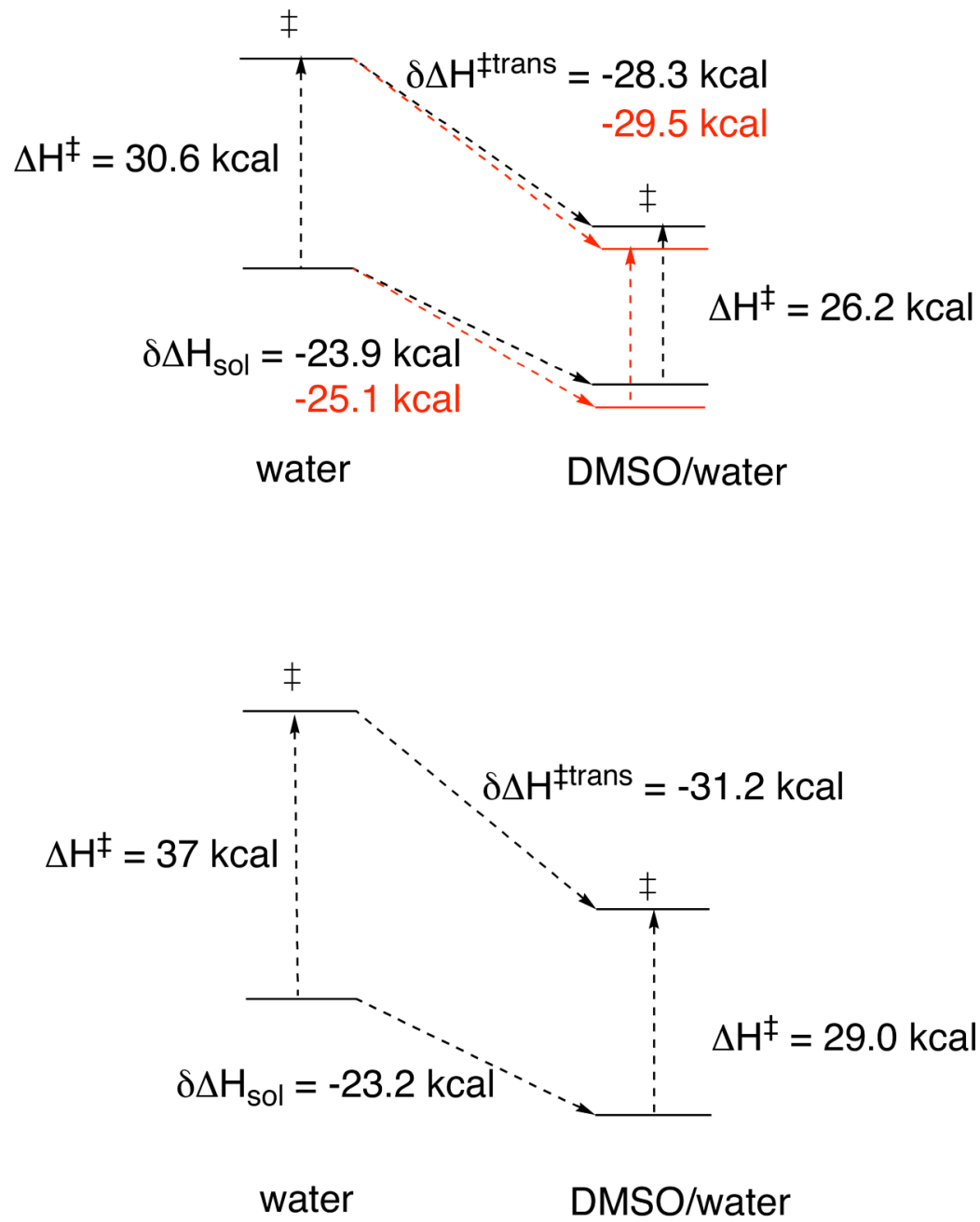

Figure 4.

Schematic representation of the enthalpies of solution of $p \mathrm{NPP}^{2-}$ (top) and $p \mathrm{NPPT}^{2-}$ (bottom), in water and in $0.6(\mathrm{~mol})$ aq DMSO, and the enthalpies of transfer for the transition states of their hydrolysis reactions in the two solvents. Red in the upper diagram indicates values if the nucleophilic water molecule is included in the treatment of the $p$ NPP reaction. 
Table 1

Enthalpies of Solution

\begin{tabular}{|c|c|c|}
\hline Compound & $\begin{array}{l}\mathrm{H}_{2} \mathrm{O} \\
\Delta \mathbf{H}_{\text {sol }}, \mathrm{kcal} / \mathrm{mol}\end{array}$ & $\begin{array}{l}\mathbf{0 . 6}(\mathrm{mol}) \mathrm{aq} \text { DMSO } \\
\Delta \mathbf{H}_{\text {sol }}, \mathrm{kcal} / \mathrm{mol}\end{array}$ \\
\hline $\begin{array}{l}{\left[(\mathrm{Bu})_{4} \mathrm{~N}\right]\left[(\mathrm{Bu})_{4} \mathrm{~B}\right]} \\
{\left[(\mathrm{Bu})_{4} \mathrm{~N}\right]_{3} \mathrm{PO}_{4}} \\
{\left[(\mathrm{Bu})_{4} \mathrm{~N}\right]_{2} \mathrm{pNPP}} \\
{\left[(\mathrm{Bu})_{4} \mathrm{~N}\right]_{2} \mathrm{PP}} \\
{\left[(\mathrm{Bu})_{4} \mathrm{~N}\right]_{2} \mathrm{PPT}}\end{array}$ & $\begin{array}{l}\text { Not Soluble } \\
-44.7 \pm 0.5 \\
-39.1 \pm 0.2 \\
-36.5 \pm 0.2 \\
-36.5 \pm 0.3\end{array}$ & $\begin{array}{l}14.0 \pm 0.3 \\
-48.3 \pm 0.3 \\
-41.4 \pm 0.3 \\
-38.1 \pm 0.3 \\
-38.1 \pm 0.5\end{array}$ \\
\hline
\end{tabular}

$a_{\text {literature value }} 9$. 
Table 2

Enthalpies of Solvent Transfer, $\delta \Delta H_{\text {sol, }}$ of Compounds and Ions (kcal/mol)

\begin{tabular}{|c|c|}
\hline Species of Interest & 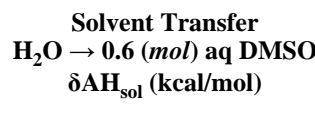 \\
\hline $\begin{array}{c}(\mathrm{Bu})_{4} \mathrm{~N}^{+a} \\
3(\mathrm{Bu})_{4} \mathrm{~N}^{+} \mathrm{PO}_{4}^{3-} \\
\mathrm{PO}_{4}^{3-} \\
2(\mathrm{Bu})_{4} \mathrm{~N}^{+} \mathrm{pNPP}^{2-} \\
\mathrm{pNPP}^{2-} \\
2(\mathrm{Bu})_{4} \mathrm{~N}^{+} \mathrm{PP}^{2-} \\
\mathrm{PP}^{2-} \\
2(\mathrm{Bu})_{4} \mathrm{~N}^{+} \mathrm{PPT}^{2-} \\
\mathrm{PPT}^{2-}\end{array}$ & $\begin{array}{c}10.8 \pm 0.1 \\
-3.56 \pm 0.91 \\
-36.0 \pm 0.9 \\
-2.3 \pm 0.4 \\
-23.9 \pm 0.3 \\
-1.6 \pm 0.4 \\
-23.2 \pm 0.4 \\
-1.6 \pm 0.5 \\
-23.2 \pm 0.6\end{array}$ \\
\hline
\end{tabular}

$a_{\text {literature value. }}{ }^{7}$ 
Table 3

Activation Parameters for Hydrolysis of $p$-NPP and $p$-NPPT in water, and in mixed solvent in which the water component is at pH 10.0, $256 \mathrm{mM}$ CAPS Buffer

\begin{tabular}{|c|c|c|c|c|}
\hline \multirow[b]{2}{*}{ Esters } & \multicolumn{2}{|c|}{$0.6(\mathrm{~mol})$ aq DMSO } & \multicolumn{2}{|c|}{$\mathrm{H}_{2} \mathrm{O}$} \\
\hline & $\Delta \mathbf{H}^{*}(\mathrm{kcal} / \mathrm{mol})$ & $\Delta S^{\dagger}(e u)$ & $\Delta \mathbf{H}^{+}(\mathrm{kcal} / \mathrm{mol})$ & $\Delta \mathbf{S}^{\ddagger}(\mathrm{eu})$ \\
\hline $\begin{array}{c}p \text {-NPP } \\
p \text {-NPPT }\end{array}$ & $\begin{array}{l}26.2 \pm 0.5 \\
29.0 \pm 0.1\end{array}$ & $\begin{array}{l}-1.5 \pm 0.5 \\
25.7 \pm 0.1\end{array}$ & $\begin{array}{c}30.6^{a} \\
37 \pm 1^{b}\end{array}$ & $\begin{array}{c}3.5^{a} \\
29 \pm 3^{b}\end{array}$ \\
\hline
\end{tabular}

